\title{
Device-to-Device Assisted Video Transmission
}

\author{
Yichao Shen, Wenwen Zhou, Peizhi Wu, Laura Toni, Pamela C. Cosman and Laurence B. Milstein \\ Department of Electrical and Computer Engineering \\ University of California, San Diego \\ La Jolla, California, USA 92093-0407 \\ Email: \{yis013,w8zhou, pew013\}@ucsd.edu, \{pcosman, milstein\}@ece.ucsd.edu, laura.toni@epfl.ch
}

\begin{abstract}
To increase spectrum efficiency, researchers envision a device-to-device (D2D) communication system in which a closely located mobile device pair may share the same spectrum with a cellular user. By opportunistically choosing the frequency, the D2D pair may increase the spectrum efficiency in terms of data rate per Hertz, at the price of additional interference to that cellular user. In previous models, users either stop cellular transmission and switch to D2D transmission or vice versa. However, if the cell is fully loaded, a D2D pair will not be able to switch back to the conventional mode because no extra resource is available. In this paper, we propose a D2D assisted model, where a D2D link is enabled to assist transmission, while keeping the conventional cellular transmission. In this way, the D2D link can be turned on and off according to the link quality. We also propose a PHY-layer study for the transmission scheme in such a way that the system throughput and the video reception quality is always improved compared to a conventional link.
\end{abstract}

\section{INTRODUCTION}

Currently, mobile video traffic is becoming one of the main drivers of cellular wireless data traffic. According to [1], the traffic generated by video delivery requests will quickly outpace mobile web content and lead to an increase in wireless data traffic by two orders of magnitude. However, the pressure it puts on the backbone cellular network is also enormous.

To address the challenge, researchers have envisioned a device-to-device (D2D) communication system to offload traffic from the central network [2]. In the literature, researchers proposed feasible ways to detect potential D2D pairs [3] [4], and to use them to transmit high quality mobile video [5]. The systems may use a dedicated radio resource or a reused resource for the D2D link [2]. In all these models, cellular transmission and D2D transmission are mutually exclusive.

In this paper, we propose a D2D assisted model, where the D2D link is enabled to assist transmission, while keeping the conventional cellular transmission. Compared to [6], we propose a more comprehensive channel model, which considers path loss, shadowing and multi-path fading. In [7], the author suggested a D2D pair may reuse frequency resource blocks from several cellular users. However, the selection and assignment is not optimal due to the computational complexity. In our model, a D2D pair will reuse the frequency with one cellular user, but that user is chosen according to an optimization criterion described in Section III. Our power control algorithm is similar to those in [8] [9], considering the signal-to-interference-and-noise ratio (SINR) degradation at the cellular receiver to control interference. However, the power control in [8] [9] is chosen to maximize the sum rate, without a constraint of how much degradation is experienced by the cellular user whose frequency is being shared, while we tradeoff the net system throughput gain with the disadvantaged cellular user's degradation.

The rest of the paper is organized as follows: Section II explains our D2D assisted model and presents evaluation metrics. Section III explains the physical layer model, including the channel model and key operations such as power control, frequency selection and adaptive MQAM. The video model is presented in Section IV. Simulation results are introduced in Section V, and Section VI draws the conclusions.

\section{SySTEM MODEL}

In this section, we present the architecture of our D2D assisted model and the evaluation metrics.

\section{A. D2D Assisted Model}

We consider a cellular network with a single base station (BS), which serves $n$ cellular users. The BS assigns orthogonal uplink and downlink frequencies to each user. Thus, in a conventional cellular transmission, a transmitter sends data to a receiver through the $\mathrm{BS}$. Among users in the cell, user A transmits video to user B, as illustrated in Fig. 1. Being in the same cell, user A and user B might be geographically close so that a direct link (i.e., D2D link) between them can be established with high quality. In this case, users A and B have the potential for D2D transmission.

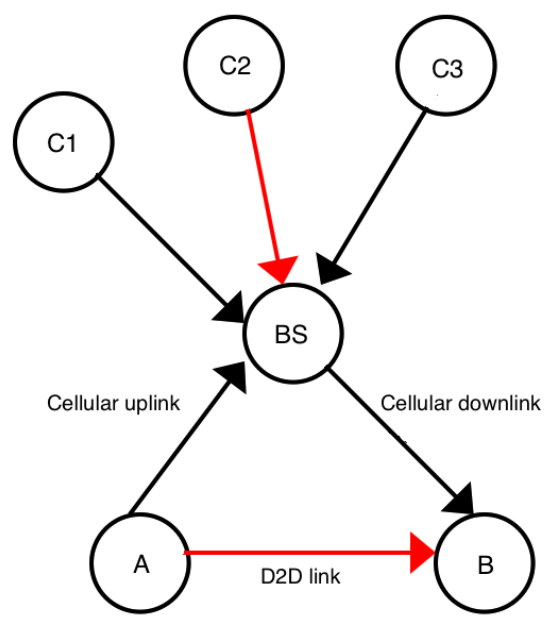

Fig. 1: D2D assisted model

When the base station notices a transmitter and its corresponding receiver are both within its domain coverage, it 
has the option of assigning the transmitter a reused frequency to initialize the D2D direct link. Since cellular downlinks are usually heavily used, the D2D pair typically shares an uplink frequency with a cellular user [3] [10]. This frequency assignment is described in Section III. The transmitter reuses the assigned frequency for the direct link, while also transmitting over the conventional link. If a drop in the quality of the D2D link is experienced, then the direct link is not used, and only the conventional transmission (i.e., the cellular one) is preserved. Our D2D assisted model thus allows for two possible parallel links. In the following, we discuss them in detail.

1) Conventional Link Model: For the conventional link, user A transmits through the conventional uplink to the $\mathrm{BS}$, and the BS forwards packets to user B through the downlink (Fig.1). Both the uplink transmitter (user A) and the downlink transmitter (the BS) have power constraints. We define the conventional link data rate: $R_{\text {conv link }}=$ $\min \left(R_{\text {conv_uplink }}, R_{\text {conv_downlink }}\right)$

2) D2D Link Model: In Fig. 1, user A has a power constraint for the D2D link as well. By using adaptive MQAM modulation, user A tries to maximize the data rate for the D2D link while attempting to maintain a pre specified level of performance. The resulting data rate is $R_{D 2 D_{-} \text {link }}$.

In the typical D2D models, where the transmitter/receiver pair may either be in the conventional mode or in the D2D mode, the D2D pair will free its dedicated uplink and downlink frequency when entering D2D mode [2] [10]. But when the D2D link quality deteriorates, it will revert to the conventional mode by asking the BS for dedicated uplink and downlink frequencies again as if they are new users in the cell. However, if the cell is extremely crowded, where no dedicated frequency is available, either this pair or another user will be dropped from the cell, which greatly impairs the user experience. In our model, we avoid this by keeping the conventional link for the pair, and adding D2D transmission to increase the throughput whenever possible. This model increases frequency efficiency in the sense that we are now transmitting more bits on the reused frequency.

Since we consider video as the underlying traffic, the D2D mode selection algorithm is carried out for every Group of Pictures (GOP) set. A GOP set contains a sufficient number of GOPs, so that the channel estimation and algorithm computation overhead is negligible. We consider a quasi-static channel such that the channel gain is constant over an entire GOP set and changes independently between GOP sets. We assume all users are experiencing small Doppler spreads, so that the receiver can learn the channel state information (CSI), and feed the CSI back to the BS for centralized decisions. The algorithm in Fig. 2 is used for every GOP set, and is decomposed into two main steps: the BS will assign a qualified D2D pair to a reused frequency and inform the D2D transmitter of its power limit, as determined by the power control. The cellular user that shares its frequency may change from one GOP set to another. But in each GOP set, that cellular user is the optimal one according to our frequency selection algorithm described below. The D2D transmitter will select a constellation size using adaptive MQAM with the given power constraint. After the direct link is established, the D2D transmitter will send the video stream over the two channels (cellular link and D2D link). Key operations such as power control, frequency selection, adaptive MQAM and frame loading are explained in Sections III and IV.

\section{B. Evaluation Metrics}

Compared to the baseline model where only the conventional mode is available, we evaluate the D2D assisted model from the following two aspects:

1) We consider the sum throughput of all the users. That is, we sum the expected number of correct bits of all the uplinks and the D2D link, and compare that value with the sum throughput of the conventional baseline model. While the D2D link leads to an improvement of the throughput, it also implies a reduction of the expected received rate for the user who shares the frequency. The latter effect is due to the co-channel interference generated by the frequency reuse. Overall, the best tradeoff is the one that leads to an improvement of the sum throughput without drastically affecting the other users.

2) We consider the video quality (measured in PSNR) at the D2D receiver, and compare the overall PSNR for this D2D assisted model with the conventional baseline model at the receiver.

\section{PHY LAYER MODEL}

In this section, we present our channel model and key operations in detail.

\section{A. Channel Model}

The channel model consists of three parts: path loss, shadow fading and multi-path fading.

1) Path Loss: According to the well-known path loss model, the received power is inversely proportional to some power of the normalized transmission distance. For $d \geq d_{0}$,

$$
P_{r}=P_{t} k\left(\frac{d_{0}}{d}\right)^{\gamma}
$$

or, in $\mathrm{dB}$,

$$
P_{r}(d B)=10 \log P_{t}+10 \log k-10 \gamma \log \left(\frac{d}{d_{0}}\right)
$$

where $k, \gamma, d_{0}$ are constants, and $d$ is the distance between the transmitter and the receiver. If $d<d_{0}$, we set $P_{r}=P_{t}$, meaning that we assume there is no path loss in this case. We take typical values from [11]: $d_{0}=10 \mathrm{~m}, \gamma=2.4, k=10^{-2.4}$.

2) Shadow Fading: Shadow fading is modeled with a lognormal distribution, and if we convert the units to $\mathrm{dB}$, then it has a Gaussian distribution [11], as in Eq. (3):

$$
p\left(\varphi_{d B}\right)=\frac{1}{\sqrt{2 \pi} \delta_{\varphi_{d B}}} \exp \left(-\frac{\left(\varphi_{d B}-\mu_{d B}\right)^{2}}{2 \delta_{\varphi_{d B}}{ }^{2}}\right)
$$

By combining the shadowing with the path loss, the average path loss $\mu_{d B}$ in Eq. (3) is zero. Also, the parameter $\delta_{\varphi_{d B}}$ in Eq. (3) is a constant, usually taken to be in the range of 4 to 12 $\mathrm{dB}$ [11]. As for the autocorrelation of the shadowing process 


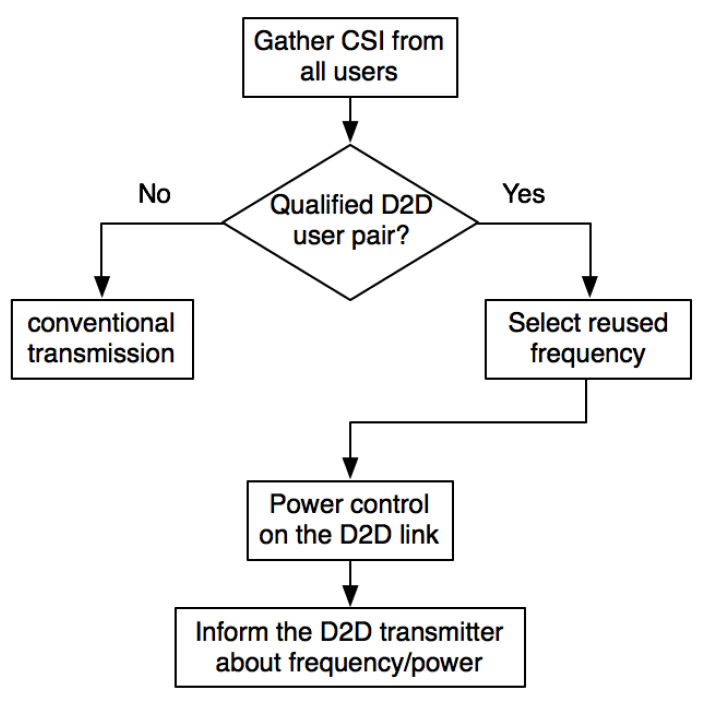

(a) Algorithm at the BS

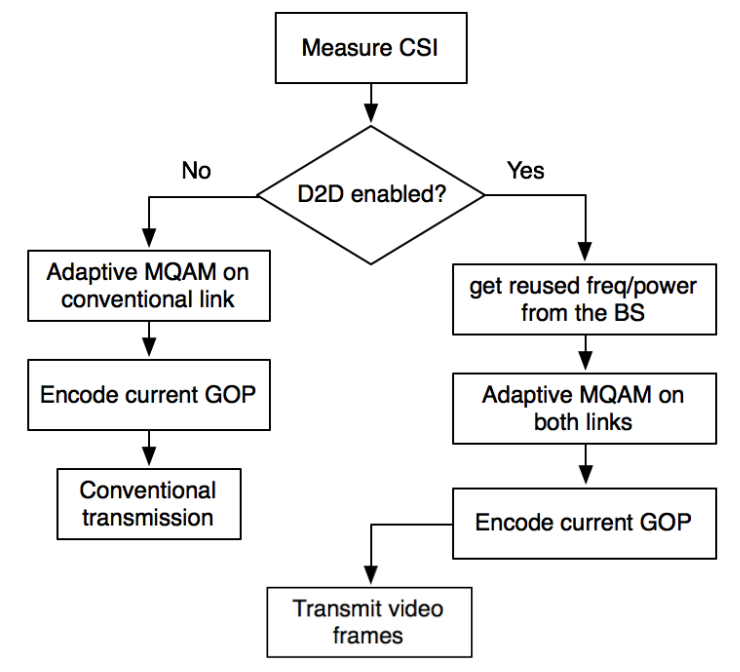

(b) Algorithm at the D2D transmitter

Fig. 2: D2D Mode Selection Algorithm for Each GOP Set

over distance, [12] assumes shadowing separated by distance $\delta$ is characterized by

$$
A\left(\delta_{x}\right)=\delta_{\varphi_{d B}}^{2} \exp \left(-\frac{\delta_{x}}{x_{c}}\right)
$$

where $x_{c}$ is the de-correlation distance, and is typically in the range from $50 \mathrm{~m}$ to $100 \mathrm{~m}$ [12]. If we combine both path loss and shadow fading, the total loss is

$$
P_{r}(d B)=10 \log P_{t}+10 \log k-10 \gamma \log \left(\frac{d}{d_{0}}\right)+\varphi_{d B}
$$

We can define channel gain as

$$
G=|g|^{2}=10^{\frac{k(d B)-10 \gamma \log \left(\frac{d}{d_{0}}\right)+\varphi_{d B}}{10}}
$$

3) Multi-path Fading: We assume the channel experiences slow Rayleigh fading, constant over an entire GOP set and changing between GOP sets. The complex attenuation is given by

$$
c=\alpha \exp (-j \theta)
$$

where $\alpha$ has a Rayleigh distribution and $\theta$ has a uniform distribution. We also assume that the coherence bandwidth equals the subcarrier bandwidth, so that the fading is independent from one subcarrier to another.

\section{B. Algorithms for Power Control and Frequency Reuse}

1) Power Control: As shown in Fig. 3, a D2D link sharing an uplink frequency results in interference to both the BS and the D2D receiver (user B). To mitigate the interference, transmission power control at the D2D transmitter is proposed to ensure that the D2D link does not jeopardize the cellular link.

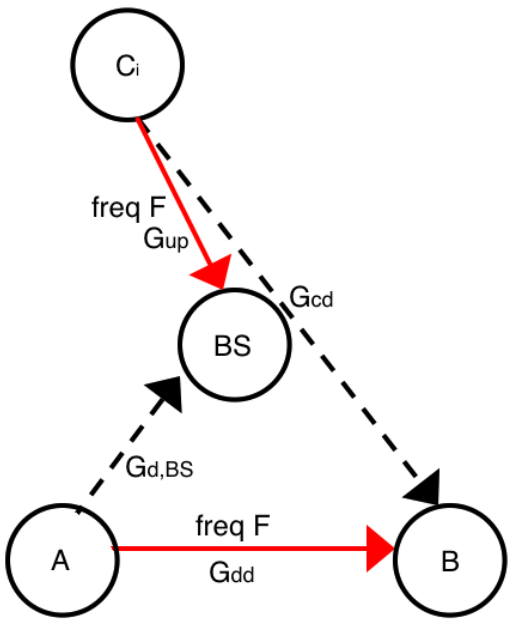

Fig. 3: By reusing the uplink frequency, the BS and the D2D receiver will suffer from interference.

The cellular user transmits to the BS through the uplink with power $P_{u p}$, and the channel gain between the cellular user and the BS is $G_{u p}$. If no D2D link shares the resource with this cellular user, there is no interference, so the SINR is

$$
S I N R_{B S}(d B)=10 \log \left(\frac{P_{u p} G_{u p}}{N W}\right)
$$

where $N$ is the power spectral density for Gaussian white noise, and $W$ is the bandwidth.

The interference to the BS from the D2D transmitter is $I_{d, B S}=P_{d d} G_{d, B S}$, where $P_{d d}$ is the transmission power at the D2D transmitter. The SINR experienced at the BS can be 
expressed as

$$
\operatorname{SINR}_{B S}{ }^{\prime}(d B)=10 \log \left(\frac{P_{u p} G_{u p}}{N W+P_{d d} G_{d, B S}}\right)
$$

We control the power $P_{d d}$ at the transmitter, so that

$$
S I N R_{B S}{ }^{\prime}(d B) \geq \eta S I N R_{B S}(d B)
$$

where $\eta$ is the interference threshold, meaning how much deterioration in SINR we allow at the cellular user receiver. From Eq. (8) to Eq. (10) we find the maximum power at the D2D transmitter A:

$$
P_{d d} \leq \frac{\left(P_{u p} G_{u p}\right)^{1-\eta}(N W)^{\eta}-N W}{G_{d, B S}}
$$

If we use the maximum allowed power to transmit on the D2D link, and denote the channel gain of the D2D link as $G_{d d}$, and the interference from the cellular uplink as $I_{c d}=P_{u p} G_{c d}$, then we obtain the SINR at the D2D receiver:

$$
S I N R_{d d}=\frac{P_{d d} G_{d d}}{N W+P_{u p} G_{c d}}
$$

Substituting $P_{d d}$ from Eq. (11) into Eq. (12), we have

$$
S I N R_{d d}=\frac{\left(\left(P_{u p} G_{u p}\right)^{1-\eta}(N W)^{\eta}-N W\right) \frac{G_{d d}}{G_{d, B S}}}{N W+P_{u p} G_{c d}}
$$

2) Selection of the Reused Frequency: We intend to reuse the frequency that maximizes the SINR at the D2D receiver, while controlling the interference at the BS by limiting the power. From the $S I N R_{d d}$ expression above, an ideal reused frequency would have the minimum gain between the cellular user and user B $\left(G_{c d}\right)$, minimum gain from user A to the BS $\left(G_{d, B S}\right)$ and maximum gain from A to B $\left(G_{d d}\right)$. Since it is not likely that a single carrier frequency will possess these three properties simultaneously, we design the algorithm to reuse that uplink frequency that maximizes Eq. (14):

$$
S I N R_{d d}(d B)=10 \log \frac{\left(\left(P_{u p} G_{u p}\right)^{1-\eta}(N W)^{\eta}-N W\right) \frac{G_{d d}}{G_{d, B S}}}{N W+P_{u p} G_{c d}}
$$

\section{Adaptive $M Q A M$}

Suppose all users employ an adaptive MQAM format. In this section, we analyze the worst-case symbol error rate (SER) for MQAM in the presence of a single interfering signal, conditioned on the channel state. Among the possible MQAM sizes, the D2D transmitter will choose the largest one such that the estimated SER is less than a predetermined threshold. The MQAM signal can be expressed as follows:

$$
\begin{aligned}
s(t) & =\sqrt{\frac{2 E_{\text {min }}}{T}} x_{c} P_{\frac{T}{2}}\left(t-\frac{T}{2}\right) \cos (2 \pi f t) \\
& +\sqrt{\frac{2 E_{\text {min }}}{T}} x_{s} P_{\frac{T}{2}}\left(t-\frac{T}{2}\right) \sin (2 \pi f t)
\end{aligned}
$$

$x_{c}, x_{s}=-L+1,-L+3 \ldots L-1, L=\sqrt{M}_{1}$, where $M_{1}$ is the MQAM modulation size for the D2D transmitter, $T$ is the symbol duration, $P_{\frac{T}{2}}()$ is a rectangular function: taking value 1 in the range of $\left[-\frac{T}{2}, \frac{T}{2}\right]$, value 0 otherwise, and $E_{\text {min }}$ is the energy of the weakest point in the constellation.
With the power constraint described in Subsection B, the transmission energy for one symbol in the in-phase branch is given by

$$
E=\int_{0}^{T}\left(\sqrt{\frac{2 E_{\text {min }}}{T}} x_{c} \cos (2 \pi f t)\right)^{2} d t=E_{\text {min }} x_{c}{ }^{2}
$$

Note that $E \leq E_{d d}$, where $E_{d d} \triangleq P_{d d} T$, and $P_{d d}$ is the power constraint. Then $E_{\min }=\frac{E_{d d}}{\left(\sqrt{M_{1}}-1\right)^{2}}$. Applying the symbol energy constraint, the D2D waveform becomes

$$
\begin{aligned}
s(t) & =\sqrt{\frac{2 E_{d d}}{T}} \frac{1}{\sqrt{M_{1}}-1} x_{c} P_{\frac{T}{2}}\left(t-\frac{T}{2}\right) \cos (2 \pi f t) \\
& +\sqrt{\frac{2 E_{d d}}{T}} \frac{1}{\sqrt{M_{1}}-1} x_{s} P_{\frac{T}{2}}\left(t-\frac{T}{2}\right) \sin (2 \pi f t)
\end{aligned}
$$

Similarly, the co-band interference from the cellular user due to the frequency reuse can be expressed as

$$
\begin{aligned}
I(t) & =\sqrt{\frac{2 E_{u p}}{T}} \frac{1}{\sqrt{M_{2}}-1} y_{c} P_{\frac{T}{2}}\left(t-\frac{T}{2}\right) \cos (2 \pi f t) \\
& +\sqrt{\frac{2 E_{u p}}{T}} \frac{1}{\sqrt{M_{2}}-1} y_{s} P_{\frac{T}{2}}\left(t-\frac{T}{2}\right) \sin (2 \pi f t)
\end{aligned}
$$

where $y_{c}, y_{s}=-\sqrt{M}_{2}+1,-\sqrt{M}_{2}+3 \ldots \bar{M}_{2}-1$, and $M_{2}$ is the MQAM modulation size for the cellular user. If the maximum power limit for all cellular users is $P_{u p}$, then the maximum symbol energy $E_{u p}=P_{u p} T$.

The transceiver block diagram for the D2D pair under the interference of a cellular user that shares the frequency with this D2D pair is shown in Fig. 4.

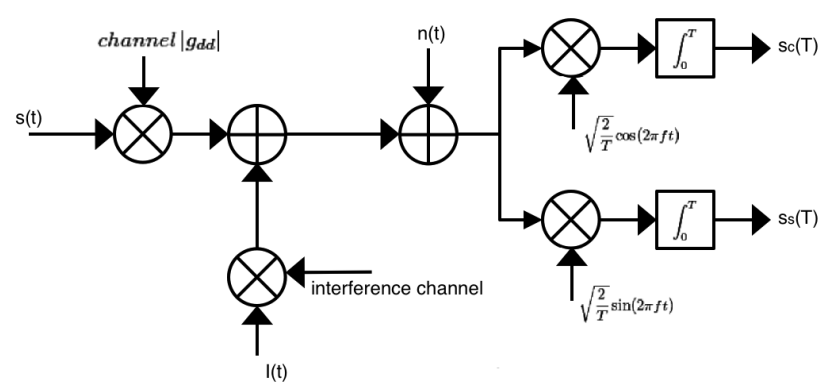

Fig. 4: Transceiver block diagram: the signal goes through the channel with additional AWGN and one interferer

After coherent demodulation, the received signal in the inphase branch is expressed as follows (the expression in the 
quadrature-phase branch is similar):

$$
\begin{aligned}
s_{c}(T) & =\sqrt{\frac{E_{d d}}{\left(\sqrt{M_{1}}-1\right)^{2}}}\left|g_{d d}\right| \underline{x_{c}} \\
& +\sqrt{\frac{E_{u p}}{\left(\sqrt{M_{2}}-1\right)^{2}}} \frac{\left|g_{c d}\right|}{T}(T-\underline{\tau}) \underline{y_{c}{ }^{0}} \cos \underline{\theta} \\
& +\sqrt{\frac{E_{u p}}{\left(\sqrt{M_{2}}-1\right)^{2}}} \frac{\left|g_{c d}\right|}{T} \underline{\tau} \underline{y}_{c}^{-1} \cos \underline{\theta} \\
& +\sqrt{\frac{E_{u p}}{\left(\sqrt{M_{2}}-1\right)^{2}}} \frac{\left|g_{c d}\right|}{T}(T-\underline{\tau}) \underline{y_{s}}{ }^{0} \sin \underline{\theta} \\
& +\sqrt{\frac{E_{u p}}{\left(\sqrt{M_{2}}-1\right)^{2}}} \frac{\left|g_{c d}\right|}{T} \underline{\tau} \underline{y s}^{-1} \sin \underline{\theta}+\underline{n_{c}(T)}
\end{aligned}
$$

where $\tau$ and $\theta$ are the delay and phase shift introduced by the channel. Interference comes from both the in-phase and quadrature-phase branch (because of the random phase), and from both the past and current symbols (because of the random time delay). In Eq. (19), $y_{c}{ }^{i}$ and $y_{s}{ }^{i}$ represent the $i$ th cellular interference MQAM symbols in the in-phase branch and the quadrature-phase branch, respectively. The noise $n_{c}(T)$ is distributed as $N\left(0, \frac{N_{0}}{2}\right)$.

As MQAM does not have a constant energy per symbol, in the worst-case, the signal of interest uses the lowest amplitude $\left(\left|x_{c}\right|=\left|x_{s}\right|=1\right)$, while all the interferer symbols correspond to the highest opposite amplitude $\left(\left|y^{0}\right|=\left|y^{-1}\right|=\sqrt{M_{2}}-1\right.$ ). If we define

$$
A_{d} \triangleq \sqrt{\frac{E_{d d}}{\left(\sqrt{M_{1}}-1\right)^{2}}}\left|g_{d d}\right|, \quad\left(\left|x_{c}\right|=\left|x_{s}\right|=1\right)
$$

and

$$
\begin{gathered}
A_{c} \triangleq-\sqrt{\frac{E_{u p}}{\left(\sqrt{M_{2}}-1\right)^{2}}}\left|g_{c d}\right|\left(-\sqrt{M_{2}}+1\right)=\sqrt{E_{u p}}\left|g_{c d}\right|, \\
\left(\left|y^{0}\right|=\left|y^{-1}\right|=\sqrt{M_{2}}-1\right)
\end{gathered}
$$

then

$$
\begin{aligned}
s_{c}(T) & =A_{d}-A_{c} \frac{T-\underline{\tau}}{T}(\cos \underline{\theta}+\sin \underline{\theta})-A_{c} \frac{\tau}{T}(\cos \underline{\theta}+\sin \underline{\theta})+\underline{n_{c}(T)} \\
& =A_{d}-\sqrt{2} A_{c} \cos \underline{\theta^{\prime}}+\underline{n_{c}(T)}, \theta^{\prime}=\theta+\frac{\pi}{4} \sim U(0,2 \pi)
\end{aligned}
$$

with a similar expression for the quadrature branch. The probability that symbol $(1,1)$ is received correctly is given by

$$
\begin{aligned}
P & =P\left(0 \leq A_{d}-\sqrt{2} A_{c} \cos \underline{\theta^{\prime}}+\underline{n_{c}(T)} \leq 2 A_{d},\right. \\
0 & \left.\leq A_{d}-\sqrt{2} A_{c} \cos \underline{\theta^{\prime}}+\underline{n_{s}(T)} \leq 2 A_{d}\right) \\
& =\left(\frac{1}{2 \pi} \int_{0}^{2 \pi} \varphi\left(\frac{A_{d}-\sqrt{2} A_{c} \cos \underline{\theta^{\prime}}}{\delta_{N}}\right)-\varphi\left(\frac{-A_{d}+\sqrt{2} A_{c} \cos \underline{\theta^{\prime}}}{\delta_{N}}\right) d \theta^{\prime}\right)^{2}
\end{aligned}
$$

Therefore, given a modulation size $M_{1}$, the corresponding estimated worst-case symbol error probability is given by $P_{S E R}=1-P$. Note that a larger MQAM modulation size will generate a higher $P_{S E R}$ value under the same channel conditions. The adaptive modulation chooses the largest MQAM size such that the estimated SER is less than the predetermined threshold.

\section{VIDEO MODEL}

For the video encoder, we use H.264 reference software JM 18.1 to compress the raw YUV video sequence. The main profile that supports the inter-coding of $\mathrm{B}$ slices is chosen, and the video is encoded as I B B P, where the GOP is one second and contains 30 frames. We use fixed 128-byte slices. The transport mechanism for the encoded video is the real time protocol (RTP).

Rate control is enabled to encode the video at different target transmission rates for both the D2D link and the cellular link. The encoder automatically sets the best quantization parameter for the first frame based on the bit rate goal and GOP length. Since the channel state is independent for every GOP set, the transmission rate constraint changes from one set to another. At the transmitter, we encode the video into 19 versions, with encoding rates from $100 \mathrm{kbps}$ to $1000 \mathrm{kbps}$, and a step size of 50kbps. After the transmission rates in the D2D link and the cellular link are determined, we can get the video source encoding rate constraint for the current GOP set, which is $R=\left(R_{\text {conv_link }}+R_{D 2 D \text { link }}\right) * R_{F E C}$, where $R_{F E C}$ is the forward error-correction (FEC). The compressed video version at the source encoding rate $R$ is fetched, coded with FEC and then partitioned to the two links according to their transmission rate.

In both the D2D link and the cellular link, the concatenation of a cyclic redundancy check (CRC) code and a rate $1 / 2$ punctured turbo code is applied to each transmitted video slice. The punctured turbo code is obtained by puncturing a rate $1 / 3$ turbo code with generator polynomial of $(13,15)_{\text {octal }}$ and puncture polynomial $(17,05,12)_{\text {octal }}$. We assume that all errors that cannot be corrected by the turbo code are detected by CRC. We generate a turbo code word from one video slice together with $16 \mathrm{CRC}$ bits.

We choose JM 16.2 for the video decoder, and slice copying is used for error concealment. Slice copying simply replaces the lost slice by the corresponding one in the previous frame. This technique has low complexity and performs well for low motion video. Then the decoded video is compared with the original video sequence to get the PSNR of the luminance component.

\section{Simulation Results}

We consider a cell size of 100 meters by 100 meters, with orthogonal carriers of bandwidth $100 \mathrm{kHz}$. The BS serves 10 cellular users and one D2D pair. The conventional link has a maximum transmission power of $100 \mathrm{~mW}$, and the D2D link transmission power is determined by the power control algorithm described in Section III. We choose a raw SER of 0.2 as the MQAM SER threshold with which to choose

2 the constellation size [13]. For each realization, we calculate the channel gains for every GOP set, and for each channel. Here, according to [14], the channel estimation overhead is less than $20 \mathrm{~ms}$. We let a GOP set contain $10 \mathrm{GOPs}$, so that the channel estimation overhead is negligible. The BS will select the best cellular user for which the D2D user can reuse uplink frequency, and will inform the D2D transmitter of its power limit. The test statistic at the receiver is given by Eq. (19). In the follows, we first determine both how much deterioration to allow at the cellular user receiver (the BS in this case), 
and what is the D2D operational distance. We quantify these parameters by testing multiple parameter sets.

\section{A. Parameter Choice}

1) Interference Threshold at the Cellular Receiver: We tested three values of the interference threshold: 10\%, 20\% and $30 \%$ decrease of SINR (in $\mathrm{dB}$ ) at the base station. We try to maximize the system throughput, while upper bounding the cellular user's degradation. Fig. 5 shows the throughput change as a function of the D2D distance. As can be seen, the D2D link always yields a positive throughput change. The data rate at the D2D link decreases, however, as D2D distance increases, which leads to a smaller system throughput gain. The reason is that the power constraint limits the maximum power at the D2D transmitter, so if the D2D pair is far apart, the link is more prone to symbol error, and thus the adaptive modulation algorithm will choose a smaller constellation size.

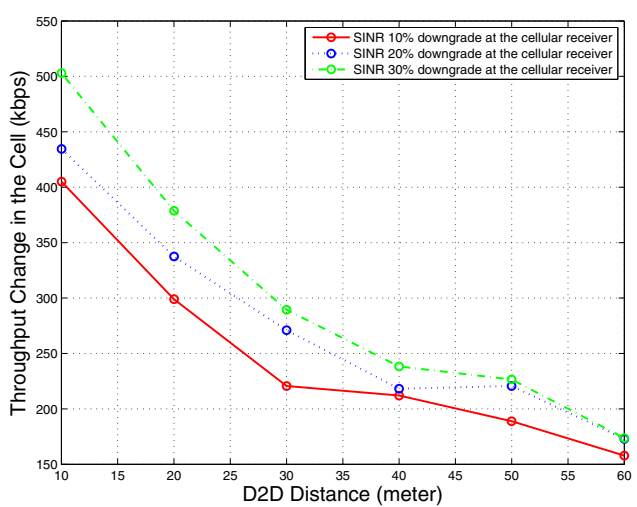

Fig. 5: Throughput change in a cell when D2D link is enabled

The cellular user that shares its uplink with the D2D pair will suffer from the interference, so we calculate the data rate change for different values of the interference threshold, while holding all the other parameters unchanged. From Fig. 6 , we see that a $10 \%$ decrease in SINR at the cellular receiver side results in an average of $8 \%$ data rate degradation, while a $30 \%$ decrease in SINR results in a $28 \%$ data rate diminution.

From Fig. 5 and Fig. 6, we see a net system throughput gain and a degraded cellular user performance for a larger interference threshold. Considering the tradeoff between these two, we are going to use a $20 \%$ decrease of SINR in $\mathrm{dB}$ at the base station as the interference threshold in the video transmission simulation described in Section V, Subsection C. Thus, at least for the parameters being used here, the tradeoff between enhanced D2D performance at the expense of degraded conventional cellular performance results in a net system gain.

2) D2D Operation Distance: For different D2D communication distance, since we are using adaptive MQAM modulation, we want to determine if the minimal alphabet size, 4QAM, results in a satisfactory video quality for the direct link. With a cell of size 100 meters by 100 meters, we consider D2D distances of $10,20,30,40,50$

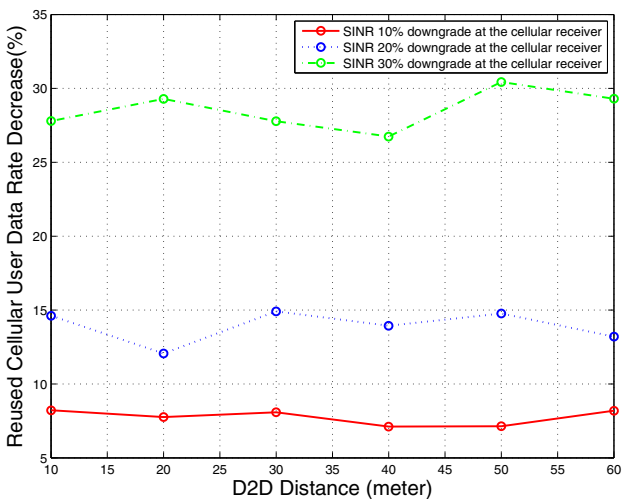

Fig. 6: Cellular data rate downgrade due to the D2D link interference

and 60 meters. Fig. 7 shows that SER increases with D2D distance. It is seen that an interference threshold of either $20 \%$ or $30 \%$ decrease in SINR at the BS will help significantly compared to a $10 \%$ threshold value.

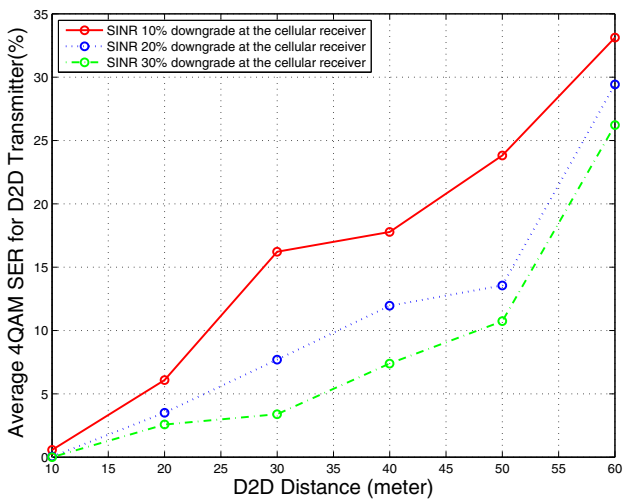

Fig. 7: 4QAM SER for the D2D link

It is also seen that, with a $20 \%$ interference threshold, when the D2D distance is below 50 meters, 4QAM (the minimum constellation size) can satisfy the raw SER threshold for constellation size selection. For the video transmission simulation below, we choose a value of 20 meters, as the D2D user separation.

\section{B. D2D Assisted Model Feasibility}

To test the system, we transmit $10^{7}$ information bits. After applying a rate $1 / 2$ turbo code, the bits are transmitted through a channel exhibiting different levels of interference. In particular, we consider three interference levels at the D2D receiver: no interference, signal-to-interference ratio (SIR) equal to $10 \mathrm{~dB}$ (low/moderate interference) and SIR equal to OdB (severe interference), in conjunction with both 4QAM and 256QAM. The performance of all the other modulation alphabet sizes should fall in the range of these two. In Fig. 8 , we show each interference level with a different line type, with circle marks representing 4QAM, and triangle marks 
representing 256QAM. For 4QAM, SIR $=10 \mathrm{~dB}$ and $\mathrm{SIR}=0 \mathrm{~dB}$ result in performance degradation by around $2 \mathrm{~dB}$ and 3 $\mathrm{dB}$, respectively, compared to the no interference curve. For 256QAM, SIR=10dB degrades the performance by $3 \mathrm{~dB}$. However, 256QAM cannot be used with SIR 0dB because of the severity of the interference.

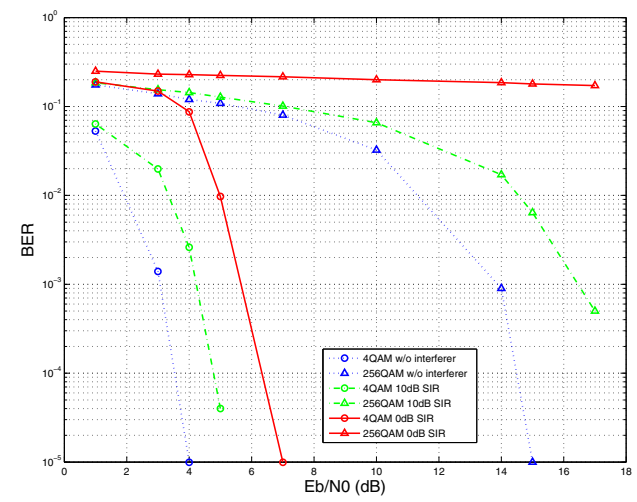

Fig. 8: BER for the D2D link under different level of interference

Note that the results shown in Fig. 8 correspond to a deterministic channel gain. However, in our D2D assisted model, channel gain is a random variable, and, when considering path loss, shadowing and multi-path fading, the PDF of such a variable is difficult to characterize. Thus, we simulated the system to obtain its performance. To obtain the average D2D channel gain, we simulated many realizations of the channel. Fig. 9 shows the Eb/N0 and SIR range for the D2D link at different D2D distances, for 300 channel realizations. From Fig. 9, we see that when the D2D distance increases, the SIR may either increase or decrease, because of the random distance change from the interferer to the receiver due to the interferer's movement. On the other hand, Eb/N0 decreases as the D2D separation increases.
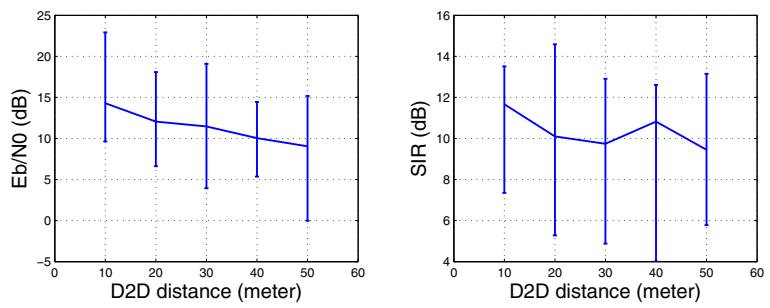

Fig. 9: Average channel gain for the D2D link

Since we chose 20 meters as D2D communication distance, we are especially interested in the channel gain at that distance. From Fig. 9, at a distance of 20 meters, the SIR ranges from $5 \mathrm{~dB}$ to $15 \mathrm{~dB}$, while the $\mathrm{Eb} / \mathrm{N} 0$ ranges from $6 \mathrm{~dB}$ to $17 \mathrm{~dB}$. Together with Fig. 8, the result suggests that in most cases, we can find an appropriate modulation scheme to transmit video information bits with a BER of $10^{-5}$.

\section{Video Reception Quality}

We tested the system with a $640 \times 480$ SDTV video. The YUV sequence is in 4:2:0 sampling format, and the frame rate is $30 \mathrm{frame} / \mathrm{s}$. The video is changing slowly, without any high-speed moving object. Recall that every GOP set experiences the independent channel fading. Also a slice is lost if there are one or more bits in error, and the bits in the lost slices are dropped, and the received video PSNR is averaged over a GOP set.

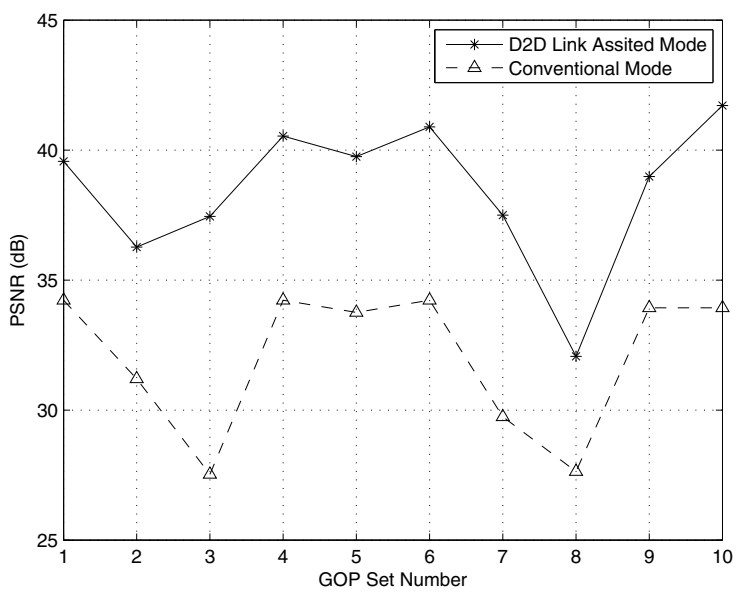

Fig. 10: Received video PSNR comparison

Fig. 10 shows the simulation results for the received video PSNR. The dashed curve indicates the PSNR in the conventional mode, and the results exhibit fair to good quality, with PSNRs ranging from $27 \mathrm{~dB}$ to $34 \mathrm{~dB}$. The solid curve is the PSNR of the received video when the D2D assisted mode is enabled, and it is seen that the PSNR is improved significantly by $8 \mathrm{~dB}$ to $10 \mathrm{~dB}$, which puts it in the good-to-excellent range of video quality. The obvious benefit that arises from the improved throughput is that the video can be compressed with higher encoding rate and hence the compression loss is reduced.

\section{CONCLUSION}

In this paper, we proposed a D2D assisted model. If two users communicating with each other are closely located, they have the potential of becoming a D2D pair by using a reused uplink frequency assigned by the base station. By employing power control and adaptive MQAM, the D2D transmitter limits the interference to that cellular user who is using the same uplink frequency during the current GOP set transmission, while maximizing its data rate on the direct link, subject to an upper bound on its transmission power. Our results showed that with the use of the D2D links, we can both increase the sum throughput in a cell, and improve the video reception quality for the D2D-enabled receiver.

\section{REFERENCES}

[1] http://www.cisco.com/en/US/solutions/collateral/ns341/ns525/ns537/ ns705/ns827/white_paper_c11-520862.html 
[2] G. Fodor, E. Dahlman, G. Mildh, S. Parkvall, N. Reider, G. Miklos, and Z. Turanyi, "Design Aspects of Network Assisted Device-to-Device Communications", IEEE Communications Magazine, May 2011.

[3] P. Janis, C.H Yu, K. Doppler, C. Ribeiro, C. Wijting, K. Hugl, O. Tirkkonen, V. Koivunen, "Device-to-Device Communication Underlaying Cellular Communications Systems", Int. J. Communications, Network and System Sciences, 2009, 3, 169-247.

[4] K. Doppler, M. Rinne, C. Wijting, C. B. Ribeiro, and K. Hugl, "Deviceto-Device Communication as an Underlay to LTE-Advanced Networks", IEEE Communications Magazine, December 2009.

[5] N. Golrezaei, A. F. Molisch, A. G. Dimakis, "Base-Station Assisted Device-to-Device Communications for High-Throughput Wireless Video Networks", IEEE International Conference on Communications, 2012.

[6] I. Forkel, M. Schinnenburg, M. Ang, "Generation of Two-Dimensional Correlated Shadowing for Mobile Radio Network Simulation", 7th International Symposium on Wireless Personal Multimedia Communications, September 2004

[7] B. Wang, L. Chen, X. Chen, X. Zhang, and D. Yang, "Resource Allocation Optimization for Device-to- Device Communication Underlaying Cellular Networks", Vehicular Technology Conference, 2011.

[8] C. Yu, O. Tirkkonen, K. Doppler and C. Ribeiro, "Power optimization of device-to-device communication underlaying cellular communication", IEEE International Conference on Communications, 2009.

[9] X. Xiao, X. Tao, J. Lu, "A QoS-Aware Power Optimization Scheme in OFDMA Systems with Integrated Device-to-Device (D2D) Communications", Vehicular Technology Conference, 2011.

[10] K. Huang, V. K. N. Lau, and Y. Chen, "Spectrum Sharing between Cellular and Mobile Ad Hoc Networks: Transmission-Capacity Tradeoff", Modeling and Optimization in Mobile, Ad Hoc, and Wireless Networks, 2009.

[11] A. Goldsmith, Wireless Communications, Cambridge University Press, 2005.

[12] M. Gudmundson, "Correlation Model for Shadow Fading in Mobile Radio System”, Elec. Lett., pp. 2145-6, November 7, 1991.

[13] D. Wang, P. C. Cosman and L. B. Milstein, "Cross Layer Resource Allocation Design for Uplink Video OFDMA Wireless Systems", IEEE Globecom, 2011.

[14] Evolved Universal Terrestrial Radio Access (E-UTRA): User Equipment (UE) radio transmission and reception (3GPP TS 36.101 version 8.18 .0 Release 8), Page 81 - 88 